\title{
Identification and Functional Analysis of the Regulatory Elements in the pHSPA6 Promoter
}

\author{
Shuyu Jiao ${ }^{1,+}{ }^{+}$, Chunyan Bai ${ }^{1,+}$, Chunyun $\mathrm{Qi}^{1}{ }^{1}$, Heyong $\mathrm{Wu}^{1}{ }^{1}$, Lanxin $\mathrm{Hu}{ }^{1}$, Feng $\mathrm{Li}^{1}{ }^{1}$, Kang Yang ${ }^{1}$, Chuheng Zhao ${ }^{1}$, \\ Hongsheng Ouyang 1,2,3,4, Daxin Pang ${ }^{1,2,3,4}$, Xiaochun Tang ${ }^{2,3,4, *}$ and Zicong Xie ${ }^{1, *}$ \\ 1 College of Animal Science, Jilin University, Changchun 130062, China; jiaosy0105@163.com (S.J.); \\ bcy@jlu.edu.cn (C.B.); qicy21@mails.jlu.edu.cn (C.Q.); heyong21@mails.jlu.edu.cn (H.W.); \\ hulx20@mails.jlu.edu.cn (L.H.); lifeng21@mails.jlu.edu.cn (F.L.); kangyang20@mails.jlu.edu.cn (K.Y.); \\ zch20@mails.jlu.edu.cn (C.Z.); ouyh@jlu.edu.cn (H.O.); pdx@jlu.edu.cn (D.P.) \\ 2 Key Lab for Zoonoses Research, Ministry of Education, Animal Genome Editing Technology Innovation \\ Center, Jilin University, Changchun 130062, China \\ 3 Chongqing Research Institute, Jilin University, Chongqing 401123, China \\ 4 Chongqing Jitang Biotechnology Research Institute Co., Ltd., Chongqing 401123, China \\ * Correspondence: xiaochuntang@jlu.edu.cn (X.T.); xzc@jlu.edu.cn (Z.X.) \\ + These authors contributed equally to this work.
}

Citation: Jiao, S.; Bai, C.; Qi, C.; Wu, H.; Hu, L.; Li, F.; Yang, K.; Zhao, C.; Ouyang, H.; Pang, D.; et al. Identification and Functional Analysis of the Regulatory Elements in the pHSPA6 Promoter. Genes 2022, 13, 189. https://doi.org/10.3390/ genes13020189

Academic Editor: Hirohide Uenishi

Received: 7 December 2021

Accepted: 19 January 2022

Published: 21 January 2022

Publisher's Note: MDPI stays neutral with regard to jurisdictional claims in published maps and institutional affiliations.

Copyright: (C) 2022 by the authors. Licensee MDPI, Basel, Switzerland. This article is an open access article distributed under the terms and conditions of the Creative Commons Attribution (CC BY) license (https:// creativecommons.org/licenses/by/ $4.0 /)$.

\begin{abstract}
Functional and expressional research of heat shock protein A6 (HSPA6) suggests that the gene is of great value for neurodegenerative diseases, biosensors, cancer, etc. Based on the important value of pigs in agriculture and biomedicine and to advance knowledge of this little-studied HSPA member, the stress-sensitive sites in porcine HSPA6 (pHSPA6) were investigated following different stresses. Here, two heat shock elements (HSEs) and a conserved region (CR) were identified in the $\mathrm{pHSPA} 6$ promoter by a CRISPR/Cas9-mediated precise gene editing strategy. Gene expression data showed that sequence disruption of these regions could significantly reduce the expression of pHSPA6 under heat stress. Stimulation studies indicated that these regions responded not only to heat stress but also to copper sulfate, MG132, and curcumin. Further mechanism studies showed that downregulated pHSPA6 could significantly affect some important members of the HSP family that are involved in HSP40, HSP70, and HSP90. Overall, our results provide a new approach for investigating gene expression and regulation that may contribute to gene regulatory mechanisms, drug target selection, and breeding stock selection.
\end{abstract}

Keywords: HSP70; pig; pHSPA6; heat shock element; stress response

\section{Introduction}

The heat shock protein 70 (HSP70) family is a set of highly conserved proteins that are known for increased synthesis, exposing organisms to a mass of cellular stresses. More recently, a vast array of post-translational modifications (PTMs) of HSP70 family proteins has been uncovered, involving altering chaperone activity, localization, and selectivity [1]. HSPA6 is a poorly characterized member of the HSP70 family and is induced after severe cellular stress [2,3]. Heat stress is not only detrimental to animal production and reproduction but also seriously damages the health of breeding animals [4]. HSPA6 was identified to have higher expression in cattle and goats under heat stress [5-7]. This may be because severe stress conditions cause HSPA6 to evolve into a gene that maintains basic biological functions [8]. Therefore, HSPA6 can be used as a candidate gene for breeding heat-resistant herds [7,9]. In addition, a previous study indicated that Parkinson's disease and Alzheimer's disease (AD) led to 30.4-fold and 6.3-fold increases, respectively, in HSPA6 expression [10-12]. HSPA6 was strongly induced by MG132 treatment of human nerve cells $[13,14]$. Knockdown experiments showed that HSPA6 could protect human nerve cells from stress [15]. Therefore, the results implied that human nerve cells treated with 
MG132 could be used as cell models for studying neurodegenerative diseases. Furthermore, HSPA6 was induced and functioned as a positive regulator for Enterovirus A71 infection, indicating that HSPA6 may assist the function of a cellular protein generally required for viral IRES activities [16]. In fact, recent studies have shown that HSPA6 is also involved in cancer progression, including that of lung cancer, triple-negative breast cancer (TNBC), and bladder cancer [17-19]. Interestingly, the HSPA6 promoter is also widely used in the biomedical field because of its ability to control cell activity at specific sites. For example, engineered $\mathrm{T}$ cells can control gene expression remotely [20]. Moreover, recent findings used the HSPA6 promoter to construct a cell line that can evaluate cell sensors in response to a stimulus based on EGFP expression [21].

HSPA 6 was cloned as a novel HSP70 gene that is different from others and expressed in many organisms [22]. There is no HSPA6 homolog in rodents, but HSPA6 exists in higher mammals, such as humans, goats, and swine $[5,23,24]$. In addition to expression among limited species, HSPA6 is barely expressed in many cells under normal culture conditions. The mRNA expression level of HSPA6 has a large increase under various cellular stresses, such as mechanical [3], copper and zinc [25], nitric oxide [26,27], heavy metal [28], and antibiotic [29] stresses. Additionally, constitutive expression of HSPA6 has been explained through the proximal promoter [30]. Deletion of the DNA fragment ( -346 to $-217 \mathrm{bp}$ ) resulted in the loss of most promoter basal activation. Furthermore, a previous assay using the DNA fragment from -287 to +110 of the HSPA6 gene (accession no. AL590385) revealed cadmium chloride-induced cytotoxicity with fourfold higher sensitivity than a cell viability test. Then, tandem repeats of the cytotoxic responding element (CRE) in the HSPA6 promoter were used to construct highly sensitive cytotoxicity sensor cells [31]. Meanwhile, some transcriptional elements were predicted, and their effect on the expression of HSPA6 was tested. AP1 plays more roles in the constitutive and inducible expression of HSPA6, whereas HSF regulates HSPA6 transcription under stress conditions [32].

After briefly summarizing the HSPA6 context, these studies mainly focused on HSPA6 promoter characterization in humans rather than pigs, which can serve as biomedical models. To further explore the pHSPA6 promoter, we identified the regulatory elements in the $\mathrm{pHSPA6}$ promoter and validated their functions based on our previously established pHSPA6-based EGFP fluorescent reporter cell line using precise gene editing strategies mediated by CRISPR/Cas9 [21].

\section{Materials and Methods}

\subsection{Cell Culture and Treatment}

Porcine primary kidney cells (PK), which were isolated from 2-day-old fetuses, porcine kidney cell lines (PK-15) (Lot Number: 58808810 ATCC Number: CCL-33), and $\mathrm{H}_{2}$ E were cultured in Dulbecco's modified Eagle's medium (DMEM) supplemented with 5\% fetal bovine serum (FBS) (Gibco, Grand Island, NY, USA). Porcine fetal fibroblasts (PFFs) were isolated from 33-day-old fetuses, and porcine alveolar macrophage cell lines (3D4/2) were obtained from Prof. Li Feng of Harbin Veterinary Research Institute, and the cells were grown in DMEM supplemented with 15\% FBS (Gibco, Grand Island, NY, USA). All cells were incubated at $37^{\circ} \mathrm{C}$ in an atmosphere of $5 \% \mathrm{CO}_{2}$, and the medium was changed every 2 days.

Cells were exposed to $42{ }^{\circ} \mathrm{C}$ for $100 \mathrm{~min}$ and recovered at $37^{\circ} \mathrm{C}$ with $5 \% \mathrm{CO}_{2}$. Concentrations of $500 \mu \mathrm{M}$ copper sulfate, $30 \mu \mathrm{M}$ MG-132, and $40 \mu \mathrm{M}$ curcumin were used to stimulate cells for $100 \mathrm{~min}$ and cultured under standard conditions (DMEM supplemented with 5\% FBS) after washing with PBS buffer solution. Finally, cells were collected at 0, 1, 2, $4,8,12$, and $24 \mathrm{~h}$ post-heat/treatment for follow-up experiments.

\subsection{Plasmids}

sgRNAs (listed in Table 1) targeting the three regions of the pHSPA6 promoter were designed by online software (http:/ / crispr.mit.edu/, accessed on 10 December 2020) and synthesized by Comate Bioscience Co., Ltd. (Changchun, China). Targeting sgRNAs were 
ligated to the PX330 vector (42230, Addgene) using the method [33] described by Zhang at the Broad Institute of MIT.

Table 1. Sequences of sgRNAs.

\begin{tabular}{ccc}
\hline Name & Primer & Sequence $\mathbf{( 5}^{\prime}-\mathbf{3}^{\prime} \mathbf{)}$ \\
\hline \multirow{2}{*}{ sgRNA-H1 } & sgRNA-H1-F & CACCGTTCGCCCGGAAGGCGCTGGA \\
& sgRNA-H1-R & AAACTCCAGCGCCTTCCGGGCGAAC \\
\hline \multirow{2}{*}{ sgRNA-H2 } & sgRNA-H2-F & CACCGTAGAACCAGCTGGGAGAAGC \\
& sgRNA-H2-R & AAACGCTTCTCCCAGCTGGTTCTAC \\
\hline \multirow{2}{*}{ sgRNA1-1 } & sgRNA1-1-F & CACCGATTGTCACAAATCCTAGTAG \\
& sgRNA1-1-R & AAACCTACTAGGATTTGTGACAATC \\
\hline \multirow{2}{*}{ sgRNA1-2 } & sgRNA1-2-F & CACCGTTAACATCATCCCTAGCCCT \\
& sgRNA1-2-R & AAACAGGGCTAGGGATGATGTTAAC \\
\hline
\end{tabular}

Four complementary sgRNA oligo DNAs were synthesized and annealed to doublestranded DNA in the presence of $10 \times$ NEB standard Taq buffer, and the whole targeting plasmids formed using this product were ligated into the BbsI sites of the vector backbone (PX330).

\subsection{Electroporation of $\mathrm{H}_{2} \mathrm{E}$ Cells}

For electroporation, approximately $30 \mu \mathrm{g}$ of plasmids and $3 \times 10^{6} \mathrm{H}_{2} \mathrm{E}$ cells were suspended in $300 \mu \mathrm{L}$ of Opti-MEM (Gibco, Grand Island, NY, USA) in $2 \mathrm{~mm}$ gap cuvettes and electroporated using specified parameters with a BTX-ECM 2001. The electroporation parameters for $\mathrm{H}_{2} \mathrm{E}$ cells were as follows: 280 volts (V), 1 millisecond (ms), and 3 pulses for 1 repeat.

\subsection{Selection of Cell Clones}

At $48 \mathrm{~h}$ posttransfection, $\mathrm{H}_{2} \mathrm{E}$ cells were inoculated into ten $100 \mathrm{~mm}$ dishes at an appropriate density (1000-2000 cells/dish). Single-cell clones were picked and inoculated in 24 -well plates. When the confluence reached $80 \%$ or more, $15 \%$ of each clone was digested and lysed with $10 \mu \mathrm{L}$ NP40 lysis buffer $(0.45 \%$ NP40 plus $0.6 \%$ proteinase K) for $1 \mathrm{~h}$ at $56{ }^{\circ} \mathrm{C}$ and $10 \mathrm{~min}$ at $95{ }^{\circ} \mathrm{C}$. The lysate was used as the PCR template and was subjected to $1 \%$ agarose gel electrophoresis. To confirm the genotype of cell clones, pLB vectors (Tiangen, Beijing, China) were combined with some PCR products, and constructors were sequenced to determine the exact sequences. Finally, the cell clones were frozen in liquid nitrogen, and the locations were recorded.

\subsection{PCR Detection}

We performed PCR to test cell clones for site-specific knockout of CR. The primers are listed in Table 2. The PCR conditions were $95^{\circ} \mathrm{C}$ for $4 \mathrm{~min} ; 94{ }^{\circ} \mathrm{C}$ for $30 \mathrm{~s}, 58{ }^{\circ} \mathrm{C}$ for $30 \mathrm{~s}$, $72{ }^{\circ} \mathrm{C}$ for $40 \mathrm{~s}$, for 32 cycles; $72{ }^{\circ} \mathrm{C}$ for $5 \mathrm{~min}$; hold at $16^{\circ} \mathrm{C}$; and the polymerase was $2 \times$ Taq PCR Mastermix (Tiangen, Beijing, China).

\subsection{Quantitative Real-Time PCR Analysis}

RZ reagent (Tiangen, Beijing, China) was used to extract total RNA. First-strand cDNAs were generated through reverse transcription using total RNA and oligo-dT primers. Porcine GAPDH served as the reference gene for the relative expression of $\mathrm{pHSPA6}$. The $2-\Delta \Delta C t$ method was performed for mRNA expression, which was standardized to the expression of endogenous U6. Primers are listed in Table 2. 
Table 2. Primers used in this study.

\begin{tabular}{|c|c|c|c|}
\hline Name & Primer & Sequence $\left(5^{\prime}-3^{\prime}\right)$ & Usage \\
\hline \multirow{2}{*}{ HSE-1-KO } & $\mathrm{H} 1-\mathrm{F}$ & СTСTCTTCСCATGGTGA & \multirow{2}{*}{ PCR } \\
\hline & $\mathrm{H} 1-\mathrm{R}$ & GCTGGTGCATCTGACTTCAT & \\
\hline \multirow{2}{*}{ HSE-2-KO } & $\mathrm{H} 2-\mathrm{F}$ & СTCTCTTCCCATGGTGA & \multirow{2}{*}{ PCR } \\
\hline & $\mathrm{H} 2-\mathrm{R}$ & GCTGGTGCATCTGACTTCAT & \\
\hline \multirow{2}{*}{ CR-KO } & F1-F & СCTTTCTGGGCTGCGACTTGAT & \multirow{2}{*}{ PCR } \\
\hline & R1-R & GGGCGGATCGTCTGTTCAAGGA & \\
\hline \multirow{2}{*}{ EGFP-KI [21] } & EGFP-KI -F & GAGGCGCATGTTCTCCAAAAACC & \multirow{2}{*}{ PCR } \\
\hline & EGFP-KI -R & AGCCACACTTGTAGTTGCACTGG & \\
\hline \multirow{2}{*}{ pHSPA6 [21] } & $\mathrm{pHSPA} 6 \mathrm{q}-\mathrm{F}$ & ATCCATGATATTGTCCTA & \multirow{2}{*}{ qPCR } \\
\hline & $\mathrm{pHSPA6} \mathrm{q}-\mathrm{R}$ & TTATGCTCTTGTTCAGTT & \\
\hline \multirow{2}{*}{ GS [21] } & GS q-F & CTTGCATCGTGTGTGCGAAG & \multirow{2}{*}{ qPCR } \\
\hline & GS q-R & GCTTAGCTTCTCGATGGCCT & \\
\hline \multirow{2}{*}{ DNAJB1 [21] } & $\mathrm{B} 1 \mathrm{q}-\mathrm{F}$ & TGACCATCGAAGTGAAGCGG & \multirow{2}{*}{ qPCR } \\
\hline & B1 q-R & TCGGCTGGAATGTTGTTGGA & \\
\hline \multirow{2}{*}{ DNAJC3 [21] } & C3 q-F & GGAGCCTGACAATGTGAATGC & \multirow{2}{*}{ qPCR } \\
\hline & C3 q-R & GACCTTCTCGAATCTGCTGGT & \\
\hline \multirow{2}{*}{$\mathrm{pHSP70.2}$} & $70 \mathrm{q}-\mathrm{F}$ & GAGCAAGGAGGAGATCGAGC & \multirow{2}{*}{ qPCR } \\
\hline & $70 \mathrm{q}-\mathrm{R}$ & GTTGAAGGCGTACGACTCCA & \\
\hline \multirow{2}{*}{$\mathrm{p} H S P 90 A A 1$} & $90 \mathrm{q}-\mathrm{F}$ & TCGAAGGGCAGTTGGAGTTC & \multirow{2}{*}{ qPCR } \\
\hline & $90 \mathrm{q}-\mathrm{R}$ & ATGAGCTCCTCGCAGTTGTC & \\
\hline
\end{tabular}

\subsection{Fluorescence Microscopy and Flow Cytometric Analysis}

These positive clones were treated with stressors at $100 \mathrm{~min}$. After $1 \mathrm{~h}$ incubation at $37^{\circ} \mathrm{C}$, cells were washed three times with PBS and then incubated with Hoechest 33342 diluted at 1 to 1000 for $6 \mathrm{~min}$ at $37^{\circ} \mathrm{C}$. After three washes with PBS, the cells were examined under a fluorescence microscope (Olympus BX51, Tokyo, Japan). The harvested cells were washed twice, resuspended in $300 \mu \mathrm{L}$ of DPBS, and analysed using a BD Accuri C6 flow cytometer.

\subsection{Statistical Analysis}

The data were statistically analyzed with GraphPad Prism software ( $t$ test), and all values were considered statistically significant at $p<0.05$.

\section{Results}

\subsection{Expression of HSPA6 in Porcine Cells}

To measure the expression of pHSPA6, we studied the effects on pHSPA6 expression in different types of porcine cells. Constitutive and inducible expression of pHSPA6 was examined in PK-15, PFF, and 3D4/2 cells at $37{ }^{\circ} \mathrm{C}$ and $42{ }^{\circ} \mathrm{C}$. Constitutive expression of pHSPA6 was detected with 2977.42-, 20,190-, and 48.87-fold increases in mRNA levels post heat stress, respectively (Figure 1a). At the same time, we analysed fluorescence intensity in EGFP-KI reporter PK-15 and PFF cells to further confirm the induction of HSPA6 in the cells (Figure 1e). Then, we detected that the expression of pHSPA6 in PK cells was significantly higher than that in PK-15 cells (Figure 1b). Next, we used PK cells for passage experiments and observed a significant reduction in F3 cells compared to the expression of pHSPA6 in F0 cells (Figure 1c). Finally, a cell density assay was performed using PK cells at F0 and three cell culture densities (30\%, 80\%, and 130\%), which showed the highest expression of pHSPA6 in low-density cell culture (30\%) and significantly reduced expression of pHSPA6 when the cell density reached $80 \%$, which may have been due to inhibition of pHSPA6 expression when the cell density exceeded $68 \%$. However, when the cell density reached 
$130 \%$, that is, the cells showed lamination, the expression of pHSPA6 did not change significantly (Figure 1d).

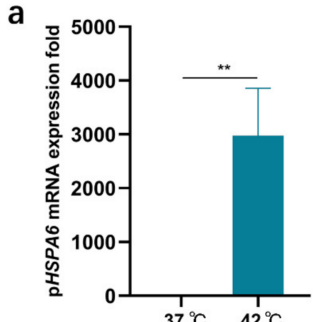

b

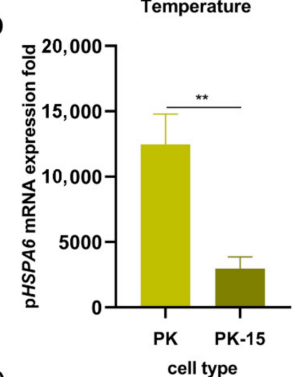

e

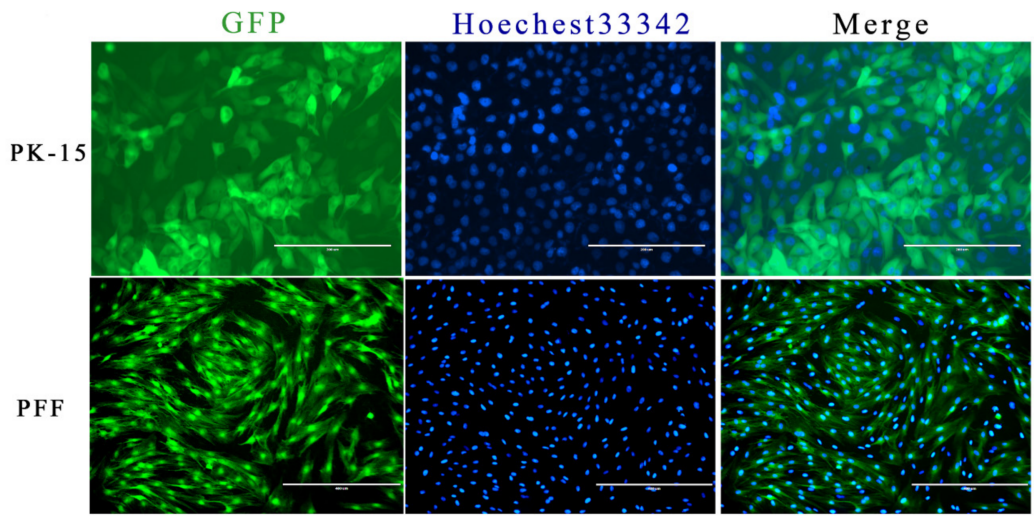

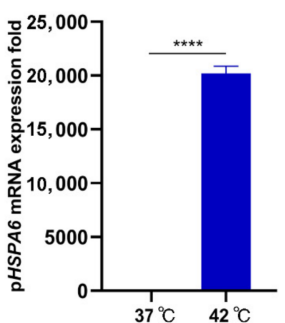

Temperature

C

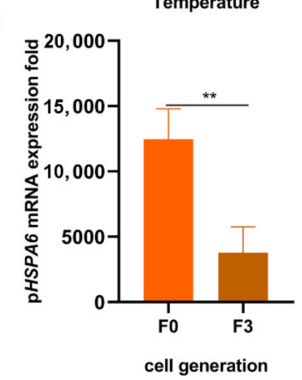

Hoechest 33342

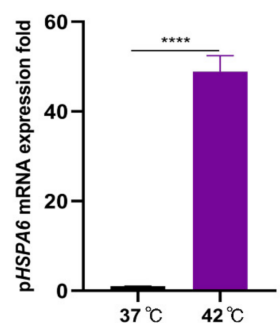

d

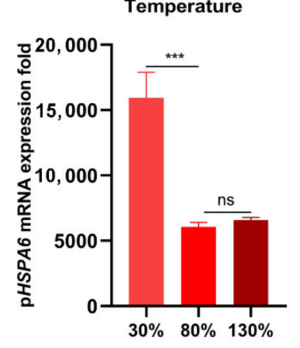

cell density

Figure 1. Analysis of the HSPA6 gene in porcine cells. (a) The mRNA expression of HSPA6 in PK-15, PFF, and 3D4/ 2 cells was detected by qPCR under heat. ${ }^{* *} p<0.01{ }^{* * * *} p<0.0001$. (b) qPCR analysis of inducible pHSPA6 expression in PK and PK-15 cells. ${ }^{* *} p<0.01$. (c) qPCR analysis of inducible pHSPA6 expression in F0 and F3 cells. ${ }^{* *} p<0.01$. (d) qPCR analysis of the cell density of inducible pHSPA6 expression in PK cells. ${ }^{* * *} p<0.001, \mathrm{~ns}=$ not significant. (e) Fluorescence microscopy analysis of the eGFP expression in PK-15 cell line and PFF cells under heat.

We concluded that cell type, cell line, generation, and cell density could impact the expression of $\mathrm{pHSPA6}$. The results indicated that $\mathrm{pHSPA6}$ was characterized by unnoticeable constitutive expression but was greatly induced following stress. We thus sought to identify key regulatory elements affecting pHSPA6 expression.

\subsection{Effect of HSE-1 on the Expression of pHSPA6}

Previous analysis for heat shock element (HSE) detection revealed a site at -100 to $-60 \mathrm{bp}$ within the human HSPA6 promoter [22,30,32]. To validate whether the site has an impact on pHSPA6 expression, we blasted its sequence against the pHSPA6 promoter and named it HSE-1; it is located at $-73 \sim-107$ bp (Figure 2a, Supplementary Figure S1a). Then, we designed a guide RNA (sgRNA-H1) to target HSE-1. Vectors combining sgRNA-H1 with Cas9 were transfected into PFFs using electroporation to assess the efficiency of sgRNAH1. After eighty-four hours, genomic DNA was isolated from these cells, and Sanger sequencing was performed using PCR products containing the target site. Chromatograms 
showed obvious multipeaks around the cleavage site. The results suggested that sgRNA-H1 could be used to effectively target HSE-1 in subsequent experiments (Figure 2b).

-195 to +48

TGAACAGACG ATCCGCCCGA ACCTTCTTCC CGAGTCAGCG GCTGAGTCAG CCAGAGGGGG CGGGCTGGGC GGCTCTAGGT TCGCCCGGAA GGCGCTGGAA GGTTCACGGT TGCGGGGGCG GGAGGAGGCG GGTCTTCCGC AACTGGATAA AAAGCTCCTG GAAGGGGAGG GAGAGCAGAT CCGAGCTGGG TGGACCGCAA GAGTGGCAGT GCTGAGCGTA CCTCCAACGT TGAGAAGCAC TAA

c

WT: GGGCGGGCTGGGCGGCTCTAGGITCGCCQGGAAGGCGCTGGAAGGTTCAC H-1: GGGCGGGCTGGGCGGCTCTAGGTTCGCCCGGAAGGCGCTTGGAAGGTTCAC H-2: GGGCGGGCTGGGCGGCTCTAGGTTCGCCCGGAAGGCGCTTGGAAGGTTCAC H-3: GGGCGGGCTGGGCGGCTCTAGGTTCGCCCGGAAGGCGCTTGGAAGGTTCAC 1-4: GGGCGGGCTGGGCGGCTCTAGGTTCGCCCGGAAGGCGCTTGGAAGGTTCAC H-5: GGGCGGGCTGGGCGGCTCTAGGTTCGCCCGGAAGGCGCTTGGAAGGTTCAC H-6: GGGCGGGCTGGGCGGCTCTAGGITCGCCCGGAAGGCGCTTGGAAGGTTCAC

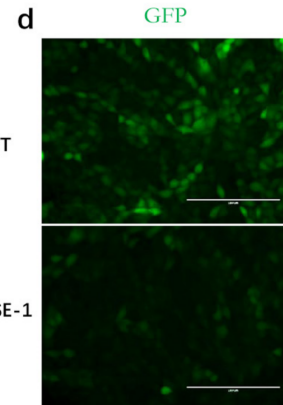
Hoechest 33342

b

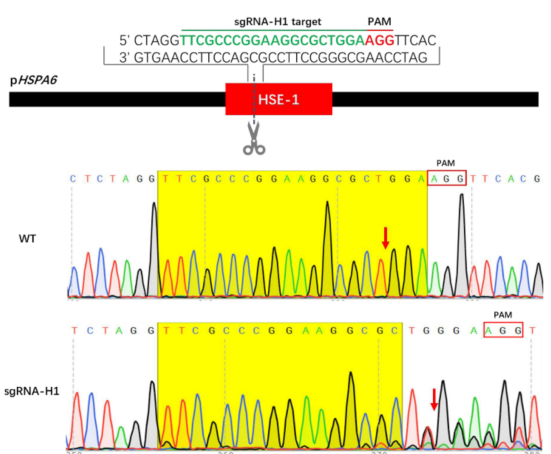
Merge
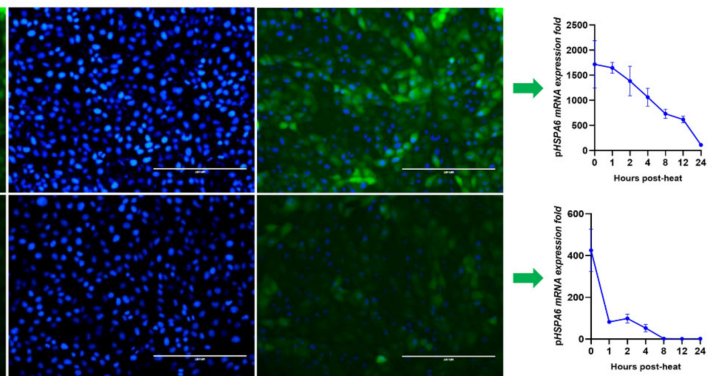

Figure 2. Effect of HSE-1 on the expression of pHSPA6. (a) The position of HSE-1 was located in the pHSPA6 promoter. (b) Strategy of sgRNA-H1 knock-in into pHSPA6; the cutting efficiency of sgRNAH1 was evaluated using chromatograms. Red blocks and red arrows represent PAMs and cleavage sites, respectively. (c) Diagram of positive clone HSE-1 after pLB vector cloning. (d) Fluorescence microscopy and qPCR analysis of pHSPA6 expression in WT and positive clone cells (data show the mean \pm S.E.M. in triplicate assay).

Previous studies used the HSPA6 promoter to construct a cell line $\left(\mathrm{H}_{2} \mathrm{E}\right)$ that can evaluate cell sensors in response to a stimulus based on EGFP expression [21]. Next, sgRNA-H1-specific PX330 plasmids were injected into $\mathrm{H}_{2} \mathrm{E}$ cells through electroporation. After forty-eight hours, we used a limiting dilution method for injecting the transfected $\mathrm{H}_{2} \mathrm{E}$ cells into $100 \mathrm{~mm}$ dishes (1500 cells per plate), and then clones were selected. The TA cloning and Sanger sequencing results were analyzed for indels, which suggested that the genotype of the HSE-1 clone was homozygous $-89 \mathrm{~T}$ ins (an insertion of ' $\mathrm{T}$ ' at $-89 \mathrm{bp}$ ) (Figure 2c; Supplementary Figure S1b).

Therefore, we speculated that the pHSPA6-89 T ins variant, which destroys an HSF binding site, determines the molecular phenotype. Furthermore, we also examined an association between the variant genotype and the fold change of pHSPA6 mRNA expression under heat stress, and the results suggested that the mutation could significantly reduce the mRNA expression level of pHSPA6 (by 75.23\%) (Figure 2d). In addition, to explore whether the pHSPA6-89 T ins variant would influence the response of pHSPA6 to other stressors, we treated wild-type and HSE-1 clone cells with copper sulfate, MG132, and curcumin. Our results indicated that the mutation resulted in $99.54 \%, 99.11 \%$, and $96.95 \%$ decreases, respectively (Supplementary Figure S1c-e). EGFP fluorescence was further confirmed via FACS analysis (Supplementary Figure S2).

\subsection{Effect of HSE-2 on the Expression of $p H S P A 6$}

A previous study predicted an HSE at -284 to -265 bp within the human HSPA6 promoter but did not explain its function [32]. To identify whether the element has an impact on the expression of pHSPA6, we blasted its sequence against the pHSPA6 promoter and named it HSE-2, which is located at -254 -235 bp (Figure 3a, Supplementary Figure S3a). 
Then, we designed a guide RNA (sgRNA-H2) to target HSE-2 and used the same method we used for HSE-1 to evaluate the targeting efficiency of sgRNA-H2. Sanger sequencing showed obvious multipeaks near the cleavage site of Cas9. Hence, sgRNA-H2 could be used to effectively target HSE-2 in subsequent experiments (Figure $3 b$ ).

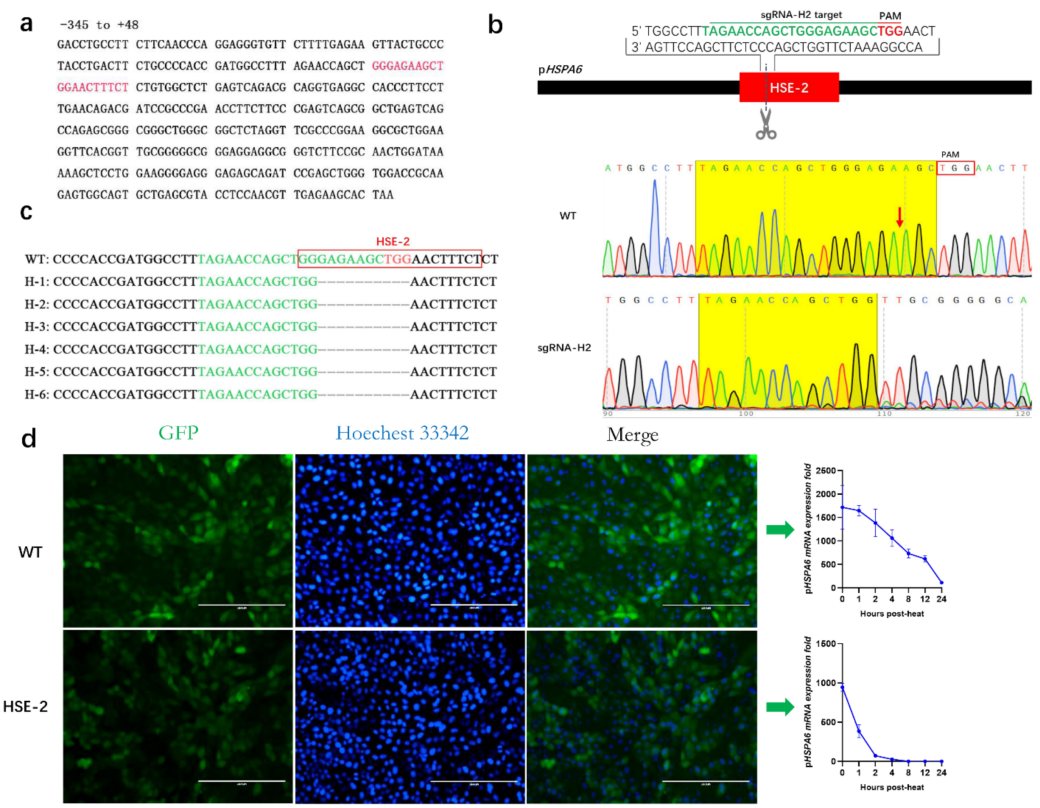

Figure 3. Effect of HSE-2 on the expression of pHSPA6. (a) The position of HSE-2 was located in the pHSPA6 promoter. (b) Strategy of sgRNA-H2 knock-in into pHSPA6; and the cutting efficiency of sgRNA-H2 was evaluated using chromatograms. Red blocks and red arrows represent PAMs and cleavage sites, respectively. (c) Diagram of positive clone HSE-2 after pLB vector cloning. (d) Fluorescence microscopy and qPCR analysis of pHSPA6 expression in WT and positive clone cells (data show the mean \pm S.E.M. in triplicate assay).

Furthermore, sgRNA-H2-specific PX330 plasmids were injected into $\mathrm{H}_{2} \mathrm{E}$ cells through electroporation, and clones were selected. The TA cloning and Sanger sequencing results were analyzed for indels, which suggested that the genotype of the HSE-2 clone was homozygous -230 10n del (10 nt deletion at -230 bp) (Figure 3c; Supplementary Figure S3b).

We thus speculated that the pHSPA6 -230 10n del variant, which destroys an HSF binding site, determines the molecular phenotype. Then, we also examined the association between the variant genotype and the fold change in pHSPA6 mRNA expression under stressors including heat, copper sulfate, MG132, and curcumin, and the results suggested that the mutation could significantly reduce the mRNA expression level of pHSPA6 (by $45.00 \%, 87.19 \%, 36.27 \%$, and 72.37\%, respectively) (Figure 3d, Supplementary Figure S3c-e). EGFP fluorescence was further confirmed via FACS analysis (Supplementary Figure S4).

\subsection{Effect of the CR on the Expression of $p H S P A 6$}

Based on homology analysis of seven species, we first found a highly conserved region (CR) located at $-646 \mathrm{bp} \sim-575 \mathrm{bp}$ within the pHSPA6 promoter (Figure $4 \mathrm{a}$ ). To detect whether CR contributes to pHSPA6 expression, we designed a pair of sgRNAs to knock out the region. Then, we used the same method we used for HSE-1 to evaluate the targeting efficiency of the pair of sgRNAs, and Sanger sequencing revealed obvious deletions between the Cas9 cleavage sites of sgRNA1-1 and sgRNA1-2. Hence, the pair of sgRNAs could be used to effectively target the CR in subsequent experiments (Figure $4 \mathrm{~b}$ ). 


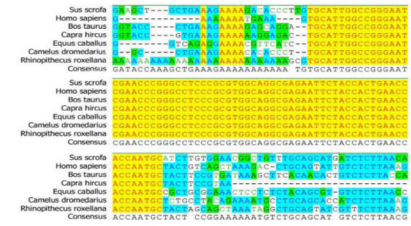

-745 to +48

CTGGTTTAT TTATGTCATT GTCACAAATC CTAGTAGGG AaAAacAaA CAAAGCTTTT TTAAATCAC TGAAGCTCCT GAAGGAAAG ACACCCTTG GCATTGGCCG GGAATCCAAC COGGGCCTCC CGCGTGGCAG GCGAGAATC TACCACTCAA CCACCAATCC ATCTTGTCGA ACGGCTGTTT GCAGCATG CTCTTAACAT CATCCCTAGC CCTAGGTCCC TTCCTAGCTG TTCTTCTCA

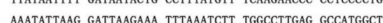
MAATATTAAG GATTAAGAA THAAATCHT TGGCTTGAG GCCATGGCTC

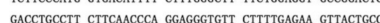

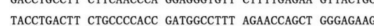
TGAACAGACG ATCCGCCCGA ACCTTCTTCC CGAGTCAGCG GCTGAGTCA CCAGAGCGGG CGGGCTGGGC GGCTCTAGGT TCGCCCGGAA GGCGCTGGA GGTTCACGGT TGCGGGGGCG GGAGGAGGCG GGTCTTCCGC AACTGGATA AAAGCTCCTG GAAGGGGAGG GAGAGCAGAT CCGAGCTGGG TGGACCGCA GAGTGGCAGT GCTGAGCGTA CCTCCAAGGT TGAGAGGCAC TAA

d
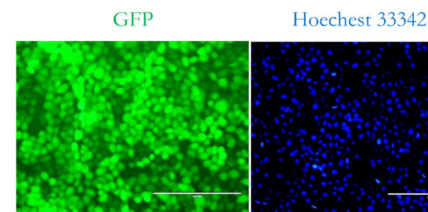
GGACTTTCT CTGTGGCTCT GAGTCAGACG CAGGTGACCC CGCCCTTO
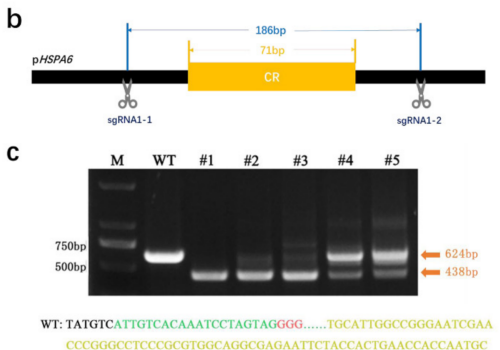
.TAACATCATCCCTAGCCCTAGGTCCCT I-1: TATGTCATIGTCACAAATCCTAG- -

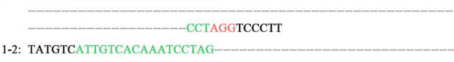

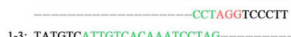

1-3: TATGTCATTGTCACAAATCCTAG-

-

- СТTAGGTCCCTI

Merge

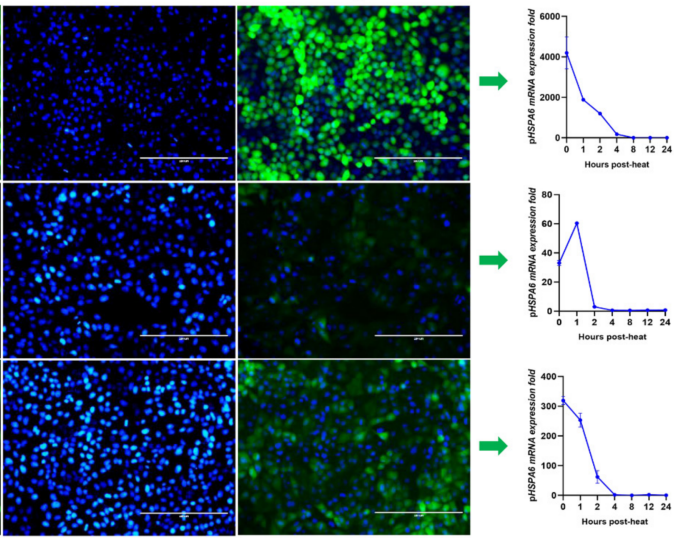

1
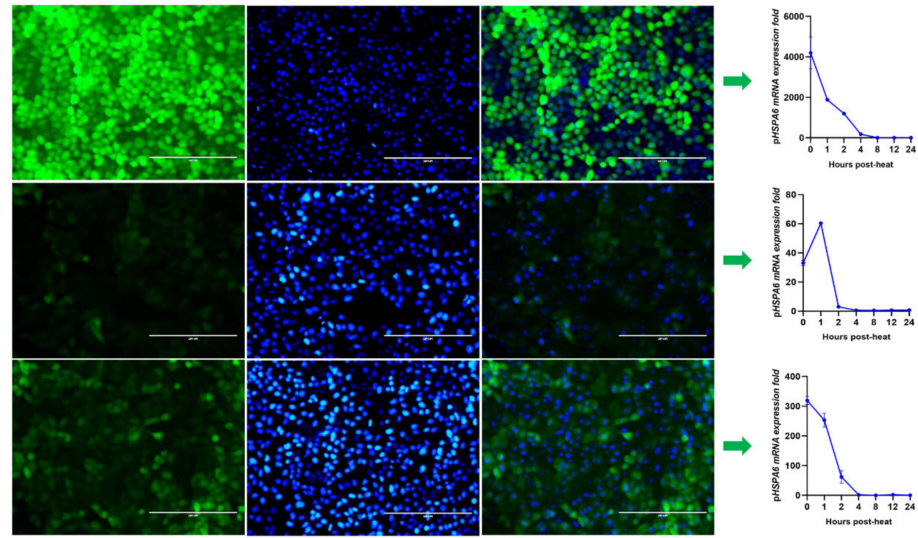

\#2

Figure 4. Effect of $C R$ on the expression of pHSPA6. (a) CR was found by blasting the HSPA6 promoter of seven species, and the position of CR was located in the pHSPA6 promoter. (b) Strategy of sgRNA11 and sgRNA1-2 knock-in into pHSPA6. (c) Selecting of positive clones using PCR. Primers are shown in Table 2; diagram of positive clone \#1 after pLB vector cloning. (d) Fluorescence microscopy and qPCR analysis of pHSPA6 expression in WT and positive clone cells (data show the mean \pm S.E.M. in triplicate assay).

Next, the pair of sgRNA-specific PX330 plasmids was injected into $\mathrm{H}_{2} \mathrm{E}$ cells through electroporation, and clones were selected. According to the results of TA cloning and Sanger sequencing, we obtained two genotypes: homozygous $-525183 \mathrm{n}$ del (183 nt deletion at $-525 \mathrm{bp}$ ) (\#1) and heterozygous -525 183n del (\#2) (Figure 4c; Supplementary Figure S5b-d). In addition, we examined the insertion of EGFP at a specific site by PCR (Supplementary Figure S5a).

Then, we also detected the fold change in pHSPA6 mRNA expression under stressors including heat, copper sulfate, MG132, and curcumin, and the results suggested that the \#1 clone could significantly reduce the mRNA expression level of pHSPA6 (by $99.21 \%, 94.85 \%$, $98.68 \%$, and $94.51 \%$, respectively) and that the \#2 clone could significantly reduce the mRNA expression level of pHSPA6 (by $92.40 \%, 94.18 \%, 91.08 \%$, and $88.66 \%$, respectively) (Figure 4d; Supplementary Figure S6a-c). EGFP fluorescence was further confirmed via FACS analysis (Supplementary Figure S7).

\subsection{Effects of the Three Regulatory Regions on the Expression of Other Genes}

A previous study confirmed that HSPA6 induction is likely to link with some members of the HSP40 family [17]. To further determine whether decreased pHSPA6 expression could alter the expression of HSP40 family members, we measured the expression of some genes related to HSPA6 in WT and clone cells. The results indicated that GS and DNAJB1 had 
a positive correlation with downregulated pHSPA6 in the three clones, and DNAJC3 had a positive correlation with downregulated pHSPA6 in clones HSE-1 and HSE-2, whereas DNAJC3 was uncorrelated with CR (Figure 5a,d-f).

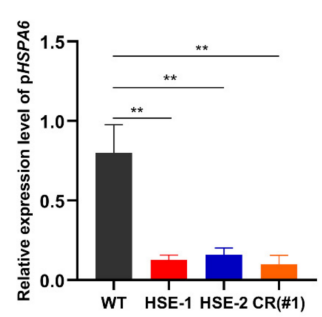

d

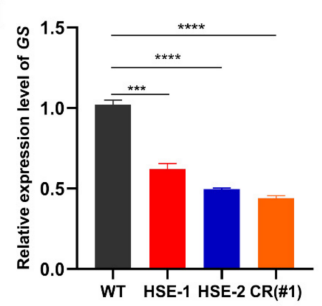

b

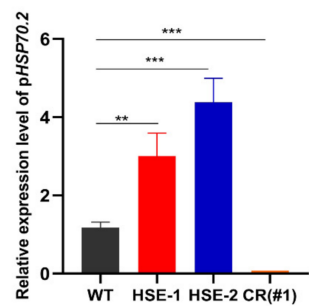

e

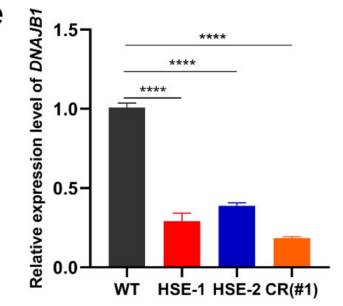

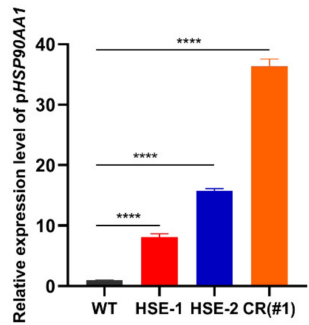

f

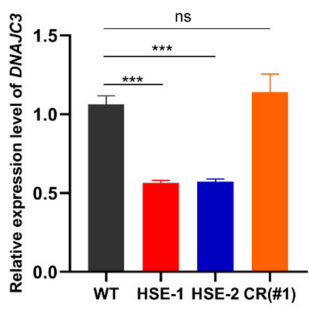

Figure 5. Effects of the three regulatory regions on the expression of other genes after thermal treatment. (a) HSE-1, HSE-2, and CR significantly reduced the expression of pHSPA6. ${ }^{* *} p<0.01$. (b) The expression of pHSP70.2 was negatively correlated with changes in pHSPA6 in clones HSE1 and HSE-2 but positively correlated with CR. ${ }^{* *} p<0.01$, ${ }^{* * *} p<0.001$. (c) The expression of $\mathrm{pHSP90AA1}$ was negatively correlated with changes in pHSPA6 in clones HSE-1, HSE-2, and CR. **** $p<0.0001$. (d) The expression of GS was positively correlated with changes in pHSPA6 in clones HSE-1, HSE-2, and CR. ${ }^{* * *} p<0.001,{ }^{* * * *} p<0.0001$. (e) The expression of DNAJB1 was positively correlated with changes in pHSPA6 in clones HSE-1, HSE-2, and CR. ${ }^{* * *} p<0.0001$. (f) The expression of DNAJC3 was positively correlated with changes in pHSPA6 in clones HSE-1 and HSE-2 but uncorrelated with CR. ${ }^{* *} p<0.001, \mathrm{~ns}=$ not significant.

Since HSP70 and HSP90 play an important role in cancer and neurodegenerative diseases, we wondered whether CRISPR/Cas9 editing of HSE-1, HSE-2, and CR affected the expression of $\mathrm{pHSP70.2}$ and $\mathrm{pHSP90AA1.} \mathrm{Therefore,} \mathrm{the} \mathrm{expression} \mathrm{levels} \mathrm{of} \mathrm{pHSPA6}$,

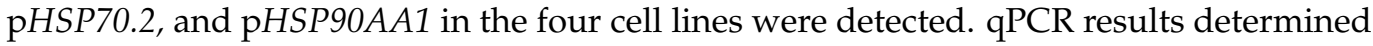
that the expression of pHSP70.2 was negatively correlated with changes in pHSPA6 in clones HSE-1 and HSE-2 but positively correlated with CR, and the expression of pHSP90AA1 was negatively correlated with changes in pHSPA6 in clones HSE-1, HSE-2, and CR (Figure 5a-c). Therefore, the expression of pHSPA6 can affect the expression of pHSP70.2 and pHSP90AA1.

\section{Discussion}

HSPA6 plays an important role in research on neurodegenerative diseases, tumors, biosensors, and other topics. Pigs have important research value in agriculture, biomedicine, and so on. Currently, most promoter studies of HSPA6 focus on humans, whereas pigs are studied less frequently. Here, we identified and verified the regulatory sequence of the pHSPA6 promoter based on $\mathrm{H}_{2} \mathrm{E}$, which can evaluate cell sensors in response to a stimulus based on EGFP expression. HSPA6 is basically not expressed in different human cells without stress, but the expression level increases rapidly after stress [34]. Consistent with the results of this study, pHSPA6 also increased significantly in different porcine cells after stress, while the increases were not consistent among cell types. This may be due to differences in the sensitivity of different types of porcine cells to the same stressor (Figure 1a). Then, we found that compared with that in porcine kidney cell lines, the expression of pHSPA6 was higher in porcine primary kidney cells after heating. The results of the passage test proved that the subgenerations were related to the expression of $\mathrm{pHSPA6}$. Previous research has shown that HSPA6 expression depends on the number of cells; therefore, we 
also performed a cell density test. The highest expression of $\mathrm{pHSPA} 6$ was detected in lowdensity cell culture (30\%), and the expression of pHSPA6 was significantly reduced when the cell density reached $80 \%$, which may have been due to pHSPA6 expression inhibition when the cell density exceeded $68 \%$. However, when the cell density reached $130 \%$, that is, the cells showed lamination, the expression of pHSPA6 did not change significantly. Hence, the results were consistent with those of a previous study [35].

The expression of HSPA6 is strictly induced, which is necessarily related to the regulatory elements in the promoter of HSPA6. Previous studies have reported that compared with other HSP70 promoters with basic expression, the upstream promoter elements, TATA box and CAAT box, of HSPA6 show nucleotide variation, and mutation analysis of these two elements indicated that they are necessary for basic expression of the human HSP70 gene [36]. In addition, it has been determined that RNA polymerase II is suspended on the HSP70 promoter under normal conditions but ceases ahead of activation with stressinduced HSF-1, leading to initiation of transcription and rapid induction [37,38]. When cells or bodies are subjected to physiological stress (such as heat), HSF1 oligomerizes to convert monomers to homotrimers, which translocate to the nucleus and bind to the HSE, thus inducing transcription of heat shock genes [39]. Two HSEs were edited by CRISPR/Cas9 technology, and our findings suggested that nucleotide variation in the two HSEs significantly changed the expression of $\mathrm{pHSPA6}$. This may be because the mutation destroys the HSE sequence and prevents HSF from binding to it; thus, it would reduce the promoter activity of HSPA6 and the expression of the gene. Interestingly, compared with the deletion of 10 bases in the HSE sequence, the insertion of a base (T) at a specific location could reduce pHSPA6 expression more. Furthermore, CR inhibited most of the promoter activity of pHSPA6, suggesting that this region holds a crucial position in the induction of pHSPA6 expression.

The promoter of HSPA6 can be used to construct biosensors based on the characteristics of strict induction. The sensitivity of the biosensor can be improved by modifying the regulatory element of the HSPA6 promoter. A previous study placed CRE and mutated AP1 in front of the HSPA6 promoter to improve sensor sensitivity for cadmium chloride [31]. HSE-1, HSE-2, and CR significantly reduced the expression of pHSPA6. We might well use these regions to improve the sensitivity of biosensors by replicating them in tandem to overexpress pHSPA6.

Various cellular stresses can greatly induce the expression of HSPA6, such as mechanical [3], copper and zinc [25], nitric oxide [26,27], heavy metal [28], and antibiotic [29] stress. MG132, as a proteasome inhibitor, can cause protein misfolding, inducing HSPA6 expression to promote protein refolding [40]. Curcumin, a root extract, has antitumor effects and can also induce HSPA6 expression. Here, cell lines were treated by heating, copper sulfate, MG132, and curcumin. The results showed that all four treatments could induce pHSPA6 expression in WT cells, which was considered the same as our previous report [21]. However, after the three regulatory sequences were edited, the expression of pHSPA6 decreased significantly, indicating that the three regulatory sequences responded not only to heat stress but also to copper sulfate, MG132, and curcumin.

HSP40, HSP70, and HSP90 have been well studied. Hsp40/DnaJ, as a co-partner, helps to achieve the complex functions of HSP70 members. The editing of three regulatory sequences in the pHSPA6 promoter can reduce the expression levels of GS, DNAJB1, and DNAJC3, but the expression of DNAJC3 was uncorrelated with the changes in pHSPA6 in clone CR. These data showed that the downregulation of pHSPA6 could affect the expression of the genes. This may be because induced HSPA6 interacts with some members of the HSP40 family [17]. HSP70 inhibitors would be a new strategy for enhancing cancer treatment due to the antiapoptotic activity of the HSP70 gene [41]. It is worth noting that these inhibitors have been hampered when used in patients due to toxicity [42]. DNAJA1 acts as a co-partner to regulate the expression of HSP70, and the study suggests that inhibition of DNAJA1 may be a novel anticancer strategy [43]. In addition, targeting the HSP40/HSP70 partner axis may also be feasible for the treatment of castration-resistant 
prostate cancer [44]. Interestingly, HSP90 is also associated with the development of a variety of cancers, but current HSP90 inhibitors do not have ideal clinical effects [45]. Therefore, HSP40s, HSP70, and HSP90 can be considered potential therapeutic targets for cancers. HSP70 and HSP90 also play an important role in neurodegenerative diseases [46, 47]. Previous studies have shown that inhibition of HSP90 can promote the expression of HSAP6 [48], but the effect of HSPA6 expression on HSP70 and HSP90 has not been studied, and the results of our study help fill this gap. Interestingly, although the editing of all three regulatory sequences reduces the expression of $\mathrm{pHSPA6}$, different regulatory regions have different effects on the expression of $\mathrm{pHSP70.2}$ and $\mathrm{pHSP90AA1}$. The expression of pHSP70.2 was negatively correlated with downregulated pHSPA6 in clones HSE-1 and HSE-2 but positively correlated with $\mathrm{CR}$, and the expression of pHSP90AA1 was negatively correlated with downregulated pHSPA6 in clones HSE-1, HSE-2, and CR (Figure 5a-c). Therefore, three regulatory regions in the $\mathrm{pHSPA} 6$ promoter are potential drug targets for the study of neurodegenerative diseases and cancer. Meanwhile, HSP70 and HSP90 family members have a significant role in animal thermotolerance $[49,50]$. Thus, these regions contribute to breeding heat-resistant herds.

\section{Conclusions}

In summary, our study reveals two HSEs and a newly discovered CR in the pHSPA6 promoter by a CRISPR/Cas9-mediated precise gene editing strategy. Gene expression data showed that sequence disruption of these regions could significantly reduce the expression of pHSPA6 under heat stress. Stimulation studies indicated that these regions responded not only to heat stress but also to copper sulfate, MG132, and curcumin. Among these regions, the knockout of CR could more strongly reduce the expression of pHSPA6 under stressors. Further mechanistic studies showed that downregulated pHSPA6 could significantly affect some important members of the HSP family that are involved in heat resistance and many diseases. Therefore, our novel results may provide a potential therapeutic strategy for neurodegenerative diseases and cancers, as well as beneficial strategies for breeding heat-resistant herds and research on biosensors.

Supplementary Materials: The following are available online at https: / www.mdpi.com/article/ 10.3390/genes13020189/s1, Figure S1: Identification of HSE-1 and its effect on pHSPA6 expression. Figure S2: EGFP fluorescence in WT and HSE-1 cells was confirmed via FACS. Figure S3: Identification of HSE-2 and its effect on pHSPA6 expression. Figure S4. EGFP fluorescence in WT and HSE-2 cells was confirmed via FACS. Figure S5: Selection of CR knockout cell clones. Figure S6: pHSPA6 expression analysis on CR under stressors. Figure S7. EGFP fluorescence in WT, \#1 and \#2 cells was confirmed via FACS.

Author Contributions: Conceptualization, Data curation, Methodology, Writing-original draft: S.J. and C.B.; Investigation, Methodology: C.Q., H.W. and L.H.; Formal Analysis: F.L., K.Y. and C.Z.; Methodology, Writing-review \& editing: H.O. and D.P.; Investigation, Writing-review \& editing, Formal analysis, Writing-original draft: X.T. and Z.X. All authors have read and agreed to the published version of the manuscript.

Funding: This work was financially supported by the National Key Research and Development Program of China Stem Cell and Translational Research (2019YFA0110702), Youth Program of the National Natural Science Foundation of China (31902229), and the Program for JLU Science and Technology Innovative Research Team (2017TD-28).

Institutional Review Board Statement: Not applicable.

Informed Consent Statement: Not applicable.

Data Availability Statement: Data are contained within the article or Supplementary Materials.

Conflicts of Interest: The authors declare no conflict of interest. 


\section{References}

1. Nitika; Porter, C.M.; Truman, A.W.; Truttmann, M.C. Post-translational modifications of Hsp70 family proteins: Expanding the chaperone code. J. Biol. Chem. 2020, 295, 10689-10708. [CrossRef]

2. Noonan, E.J.; Place, R.F.; Giardina, C.; Hightower, L.E. Hsp70B' regulation and function. Cell Stress Chaperon. 2007, 12, 219-229. [CrossRef]

3. Xu, Q.; Schett, G.; Li, C.; Hu, Y.; Wick, G. Mechanical stress-induced heat shock protein 70 expression in vascular smooth muscle cells is regulated by Rac and Ras small G proteins but not mitogen-activated protein kinases. Circ. Res. 2000, 86, 1122-1128. [CrossRef]

4. Hyder, I.; Sejian, V.; Bhatta, R.; Gaughan, J.B. Biological role of melatonin during summer season related heat stress in livestock. Biol. Rhythm. Res. 2017, 48, 297-314. [CrossRef]

5. Banerjee, D.; Upadhyay, R.C.; Chaudhary, U.B.; Kumar, R.; Singh, S.; Ashutosh, G.J.M.; Polley, S.; Mukherjee, A.; Das, T.K.; De, S. Seasonal variation in expression pattern of genes under HSP70. Cell Stress Chaperon. 2014, 19, 401-408. [CrossRef] [PubMed]

6. Mohanarao, G.J.; Mukherjee, A.; Banerjee, D.; Gohain, M.; Dass, G.; Brahma, B.; Datta, T.K.; Upadhyay, R.C.; De, S. HSP70 family genes and HSP27 expression in response to heat and cold stress in vitro in peripheral blood mononuclear cells of goat (Capra hircus). Small Rumin. Res. 2014, 116, 94-99. [CrossRef]

7. Rocha, R.; Baena, M.M.; Estopa, A.D.C.; Gervásio, I.C.; Ibelli, A.M.G.; Gionbelli, T.R.S.; Gionbelli, M.P.; De Freitas, R.T.F.; Meirelles, S.L.C. Differential expression of HSF1 and HSPA6 genes and physiological responses in Angus and Simmental cattle breeds. J. Therm. Biol. 2019, 84, 92-98. [CrossRef]

8. Hageman, J.; Van Waarde-Verhagen, M.; Zylicz, A.; Walerych, D.; Kampinga, H.H. The diverse members of the mammalian HSP70 machine show distinct chaperone-like activities. Biochem. J. 2011, 435, 127-142. [CrossRef] [PubMed]

9. Hyder, I.; Pasumarti, M.; Reddy, P.R.; Prasad, C.S.; Kumar, K.A.; Sejian, V. Thermotolerance in Domestic Ruminants: A HSP70 Perspective. In Heat Shock Proteins and Stress; Springer: Singapore, 2017; Volume 12, pp. 3-35.

10. Santpere, G.; Esparcia, P.G.; Benito, P.A.; Lorente-Galdos, B.; Navarro, A.; Ferrer, I. Transcriptional network analysis in frontal cortex in Lewy body diseases with focus on dementia with Lewy bodies. Brain Pathol. 2018, 28, 315-333. [CrossRef]

11. Henderson-Smith, A.; Corneveaux, J.J.; De Both, M.; Cuyugan, L.; Liang, W.S.; Huentelman, M.; Adler, C.; Driver-Dunckley, E.; Beach, T.G.; Dunckley, T.L. Next-generation profiling to identify the molecular etiology of Parkinson dementia. Neurol. Genet. 2016, 2, e75. [CrossRef] [PubMed]

12. Annese, A.; Manzari, C.; Lionetti, C.; Picardi, E.; Horner, D.S.; Chiara, M.; Caratozzolo, M.F.; Tullo, A.; Fosso, B.; Pesole, G.; et al. Whole transcriptome profiling of Late-Onset Alzheimer's Disease patients provides insights into the molecular changes involved in the disease. Sci. Rep. 2018, 8, 4282. [CrossRef]

13. Becirovic, L.; Brown, I.R. Targeting of Heat Shock Protein HSPA6 (HSP70B') to the Periphery of Nuclear Speckles is Disrupted by a Transcription Inhibitor Following Thermal Stress in Human Neuronal Cells. Neurochem. Res. 2016, 42, 406-414. [CrossRef]

14. Deane, C.; Brown, I.R. Differential Targeting of Hsp70 Heat Shock Proteins HSPA6 and HSPA1A with Components of a Protein Disaggregation/Refolding Machine in Differentiated Human Neuronal Cells following Thermal Stress. Front. Neurosci. 2017, 11, 227. [CrossRef] [PubMed]

15. Deane, C.; Brown, I.R. Knockdown of Heat Shock Proteins HSPA6 (Hsp70B') and HSPA1A (Hsp70-1) Sensitizes Differentiated Human Neuronal Cells to Cellular Stress. Neurochem. Res. 2017, 43, 340-350. [CrossRef]

16. Su, Y.-S.; Hwang, L.-H.; Chen, C.-J. Heat Shock Protein A6, a Novel HSP70, Is Induced During Enterovirus A71 Infection to Facilitate Internal Ribosomal Entry Site-Mediated Translation. Front. Microbiol. 2021, 12, 664955. [CrossRef]

17. Wang, L.; Hou, J.; Wang, J.; Zhu, Z.; Zhang, W.; Zhang, X.; Shen, H.; Wang, X. Regulatory roles of HSPA6 in Actinidia chinensis Planch. root extract (acRoots)-inhibited lung cancer proliferation. Clin. Transl. Med. 2020, 10, e46. [CrossRef]

18. Shen, S.; Wei, C.; Fu, J. RNA-Sequencing Reveals Heat Shock 70-kDa Protein 6 (HSPA6) as a Novel Thymoquinone-Upregulated Gene That Inhibits Growth, Migration, and Invasion of Triple-Negative Breast Cancer Cells. Front. Oncol. 2021, $11,667995$. [CrossRef]

19. Shin, S.-S.; Song, J.-H.; Hwang, B.; Noh, D.-H.; Park, S.L.; Kim, W.T.; Kim, W.-J.; Moon, S.-K. HSPA6 augments garlic extractinduced inhibition of proliferation, migration, and invasion of bladder cancer EJ cells; Implication for cell cycle dysregulation, signaling pathway alteration, and transcription factor-associated MMP-9 regulation. PLoS ONE 2017, 12, e0171860. [CrossRef] [PubMed]

20. Miller, I.C.; Castro, M.G.; Maenza, J.; Weis, J.P.; Kwong, G.A. Remote Control of Mammalian Cells with Heat-Triggered Gene Switches and Photothermal Pulse Trains. ACS Synth. Biol. 2018, 7, 1167-1173. [CrossRef] [PubMed]

21. Xie, Z.; Sun, R.; Qi, C.; Jiao, S.; Jiang, Y.; Liu, Z.; Zhao, D.; Liu, R.; Li, Q.; Yang, K.; et al. Generation of a pHSPA6 gene-based multifunctional live cell sensor. Biochim. Biophys. Acta (BBA) Bioenerg. 2021, 1868, 118919. [CrossRef]

22. Leung, T.K.; Rajendran, M.Y.; Monfries, C.; Hall, C.; Lim, L. The human heat-shock protein family. Expression of a novel heat-inducible HSP70 (HSP70B') and isolation of its cDNA and genomic DNA. Biochem. J. 1990, 267, 125-132. [CrossRef]

23. Dezeure, F.; Vaiman, M.; Chardon, P. Characterization of a polymorphic heat shock protein 70 gene in swine outside the SLA major histocompatibility complex. Biochim. Biophys. Acta (BBA) Gene Struct. Expr. 1993, 1174, 17-26. [CrossRef]

24. Parsian, A.J.; Sheren, J.E.; Tao, T.Y.; Goswami, P.; Malyapa, R.; Van Rheeden, R.; Watson, M.S.; Hunt, C.R. The human Hsp70B gene at the HSPA7 locus of chromosome 1 is transcribed but non-functional. Biochim. Biophys. Acta (BBA) Gene Struct. Expr. 2000, 1494, 201-205. [CrossRef] 
25. Hatayama, T.; Asai, Y.; Wakatsuki, T.; Kitamura, T.; Imahara, H. Regulation of hsp70 Synthesis Induced by Cupric Sulfate and Zinc Sulfate in Thermotolerant HeLa Cells. J. Biochem. 1993, 114, 592-597. [CrossRef] [PubMed]

26. Byrne, C.R.; Hanson, P.J. Induction of heat shock protein 72 by a nitric oxide donor in guinea-pig gastric mucosal cells. Eur. J. Pharmacol. 1998, 353, 117-122. [CrossRef]

27. Xu, Q.; Hu, Y.; Kleindienst, R.; Wick, G. Nitric oxide induces heat-shock protein 70 expression in vascular smooth muscle cells via activation of heat shock factor 1. J. Clin. Investig. 1997, 100, 1089-1097. [CrossRef] [PubMed]

28. Levinson, W.; Oppermann, H.; Jackson, J. Transition series metals and sulfhydryl reagents induce the synthesis of four proteins in eukaryotic cells. Biochim. Biophys. Acta (BBA) Nucleic Acids Protein Synth. 1980, 606, 170-180. [CrossRef]

29. Romano, C.; Benedetto, N.; Catania, M.; Rizzo, A.; Gallè, F.; Losi, E.; Hasty, D.; Rossano, F. Commonly used antibiotics induce expression of Hsp 27 and Hsp 60 and protect human lymphocytes from apoptosis. Int. Immunopharmacol. 2004, 4, 1067-1073. [CrossRef]

30. Rohmer, S.; Mainka, A.; Knippertz, I.; Hesse, A.; Nettelbeck, D.M. Insulated hsp70B' promoter: Stringent heat-inducible activity in replication-deficient, but not replication-competent adenoviruses. J. Gene Med. 2008, 10, 340-354. [CrossRef]

31. Wada, K.-I.; Taniguchi, A.; Okano, T. Highly sensitive detection of cytotoxicity using a modified HSP70B' promoter. Biotechnol. Bioeng. 2007, 97, 871-876. [CrossRef]

32. Ramirez, V.P.; Stamatis, M.; Shmukler, A.; Aneskievich, B.J. Basal and stress-inducible expression of HSPA6 in human keratinocytes is regulated by negative and positive promoter regions. Cell Stress Chaperon. 2014, 20, 95-107. [CrossRef] [PubMed]

33. Ran, F.A.; Hsu, P.D.; Lin, C.-Y.; Gootenberg, J.S.; Konermann, S.; Trevino, A.E.; Scott, D.A.; Inoue, A.; Matoba, S.; Zhang, Y.; et al. Double Nicking by RNA-Guided CRISPR Cas9 for Enhanced Genome Editing Specificity. Cell 2013, 154, 1380-1389. [CrossRef]

34. Chow, A.M.; Mok, P.; Xiao, D.; Khalouei, S.; Brown, I.R. Heteromeric complexes of heat shock protein 70 (HSP70) family members, including Hsp70B', in differentiated human neuronal cells. Cell Stress Chaperon. 2010, 15, 545-553. [CrossRef] [PubMed]

35. Noonan, E.J.; Place, R.F.; Rasoulpour, R.J.; Giardina, C.; Hightower, L.E. Cell number-dependent regulation of Hsp70B' expression: Evidence of an extracellular regulator. J. Cell. Physiol. 2007, 210, 201-211. [CrossRef] [PubMed]

36. Greene, J.M.; Larin, Z.; Taylor, I.C.; Prentice, H.; A Gwinn, K.; E Kingston, R. Multiple basal elements of a human hsp70 promoter function differently in human and rodent cell lines. Mol. Cell. Biol. 1987, 7, 3646-3655. [CrossRef]

37. Giardina, C.; Pérez-Riba, M.; Lis, J.T. Promoter melting and TFIID complexes on Drosophila genes in vivo. Genes Dev. 1992, 6, 2190-2200. [CrossRef]

38. Lis, J.; Wu, C. Protein traffic on the heat shock promoter: Parking, stalling, and trucking along. Cell 1993, 74, 1-4. [CrossRef]

39. Tang, H.; Liu, Y.; Madabusi, L.; Gilmour, D.S. Promoter-Proximal Pausing on the hsp70 Promoter in Drosophila melanogaster Depends on the Upstream Regulator. Mol. Cell. Biol. 2000, 20, 2569-2580. [CrossRef]

40. Deane, C.A.; Brown, I.R. Intracellular Targeting of Heat Shock Proteins in Differentiated Human Neuronal Cells Following Proteotoxic Stress. J. Alzheimer's Dis. 2018, 66, 1295-1308. [CrossRef]

41. Tukaj, S. Heat Shock Protein 70 as a Double Agent Acting Inside and Outside the Cell: Insights into Autoimmunity. Int. J. Mol. Sci. 2020, 21, 5298. [CrossRef]

42. Evans, C.G.; Chang, C.Y.; Gestwicki, J.E. Heat Shock Protein 70 (Hsp70) as an Emerging Drug Target. J. Med. Chem. 2010, 53, 4585-4602. [CrossRef] [PubMed]

43. Nitika; Blackman, J.S.; Knighton, L.E.; Takakuwa, J.E.; Calderwood, S.K.; Truman, A.W. Chemogenomic screening identifies the Hsp70 co-chaperone DNAJA1 as a hub for anticancer drug resistance. Sci. Rep. 2020, 10, 13831. [CrossRef] [PubMed]

44. Moses, M.A.; Kim, Y.S.; Rivera-Marquez, G.M.; Oshima, N.; Watson, M.J.; Beebe, K.E.; Wells, C.; Lee, S.; Zuehlke, A.D.; Shao, H.; et al. Targeting the Hsp40/Hsp70 Chaperone Axis as a Novel Strategy to Treat Castration-Resistant Prostate Cancer. Cancer Res. 2018, 78, 4022-4035. [CrossRef]

45. Tukaj, S.; Węrzyn, G. Anti-Hsp90 therapy in autoimmune and inflammatory diseases: A review of preclinical studies. Cell Stress Chaperon. 2016, 21, 213-218. [CrossRef] [PubMed]

46. Hu, F.; Zhou, J.; Lu, Y.; Guan, L.; Wei, N.-N.; Tang, Y.-Q.; Wang, K. Inhibition of Hsp70 Suppresses Neuronal Hyperexcitability and Attenuates Epilepsy by Enhancing A-Type Potassium Current. Cell Rep. 2019, 26, 168-181.e4. [CrossRef]

47. Luo, W.; Sun, W.; Taldone, T.; Rodina, A.; Chiosis, G. Heat shock protein 90 in neurodegenerative diseases. Mol. Neurodegener. 2010, 5, 24. [CrossRef]

48. Kuballa, P.; Baumann, A.-L.; Mayer, K.; Bär, U.; Burtscher, H.; Brinkmann, U. Induction of heat shock protein HSPA6 (HSP70B') upon HSP90 inhibition in cancer cell lines. FEBS Lett. 2015, 589, 1450-1458. [CrossRef]

49. Badri, T.M.; Chen, K.L.; Alsiddig, M.A.; Li, L.; Cai, Y.; Wang, G.L. Genetic polymorphism in Hsp90AA1 gene is associated with the thermotolerance in Chinese Holstein cows. Cell Stress Chaperon. 2018, 23, 639-651. [CrossRef]

50. Hassan, F.; Nawaz, A.; Rehman, M.S.; Ali, M.A.; Dilshad, S.M.; Yang, C. Prospects of HSP70 as a genetic marker for thermotolerance and immuno-modulation in animals under climate change scenario. Anim. Nutr. 2019, 5, 340-350. [CrossRef] 